\title{
The Galactic Halo from Microlensing
}

\author{
Alain Milsztajn (amilsztajn@cea.fr) \\ DSM-DAPNIA-Service de Physique des Particules, CEA-Saclay, \\ F-91191-Gif-sur-Yvette, France
}

\begin{abstract}
.
The status of the microlensing search for galactic dark matter in the form of massive astronomical compact halo objects (machos) is reviewed. Unresolved issues are discussed, as well as possible ways to solve these.
\end{abstract}

\section{Introduction}

The mass of our Galaxy can be computed from dynamical studies of its rotation and of the motion of its satellites, or alternatively it can be evaluated from its visible components, primarily stars. That these two estimates disagree by a factor 5-10 constitutes the problem of Galactic dark matter. Either the laws of dynamics we use are wrong on galactic scales (few kiloparsecs), or there exists some form of galactic matter that does not emit or absorb enough electromagnetic radiation to be directly detectable. Studies of many other spiral galaxies confirm that this problem is not unique to the Milky Way.

Originally proposed by B. Paczyński (1986) as a probe of galactic dark matter, the gravitational microlensing technique relies on detecting the transient magnification and/or deflection of the light from extragalactic stars by intervening machos. The a priori mass range for machos is very wide, between about $10^{-7}$ and $10^{4} M_{\odot}$ (corresponding to event durations ranging from one hour to a few decades). Lighter primordial $\mathrm{H} / \mathrm{He}$ objects would have evaporated since the galaxy formed (de Rujula et al., 1992); a halo full of heavier objects would be devoid of globular clusters by now (Arras and Wasserman, 1999).

The search for gravitational microlensing phenomena in our Galaxy has now been going on for over a decade. Although the prime suspects for machos were initially brown dwarfs $\left(0.01\right.$ to $\left.0.1 M_{\odot}\right)$, and although many arguments existed against real or imaginary objects of other masses, the survey groups have chosen to cover the widest possible fraction of the 11 orders of magnitude macho mass range. The first microlensing candidates were reported in 1993, towards the LMC (Alcock et al., 1993; Aubourg et al., 1993) and the Galactic Centre (Udalski et al., 1993) by the EROS, MACHO and OGLE collaborations. As the present review centers on halo dark matter, I will mostly discuss 
results from the former two groups. (OGLE microlensing studies have concentrated on the Galactic Bulge, see e.g. Udalski et al., 2000.)

Other groups contribute to the field. Three of them are follow-up groups, GMAN, MPS and PLANET; they observe microlensing events alerted upon by the survey groups. The MOA group conducts a survey mainly dedicated to the search for planets (see e.g. Bond et al., 2001), a topic that is also a prime motivation of MPS (Rhie et al., 2000) and PLANET (Albrow et al., 2000). Finally, the POINT-AGAPE group attacks the more ambitious goal of microlensing towards M31 (Aurière et al., 2001).

As the present review is short and contains no calculations, I refer the interested reader to other sources (Paczyński, 1996; Roulet and Möllerach, 1997; Gould, 2000; Gould, 2001).

This article is organised as follows. In Sect. 2, I recall the main properties of microlensing and the information that can be obtained from its observation. Sect. 3 discusses results from observations towards the Large Magellanic Cloud (LMC). In Sect. 4, the same is done for the Small Magellanic Cloud (SMC). Sect. 5 contains a critical assesment of these results, and conjectures about what the future may hold.

Finally, Sect. 6 gives my conclusions.

\section{Properties of Microlensing}

\subsection{Simple Microlensing}

When both the source star and the lens are simple (non-binary) and their size can be neglected, microlensing depends on two distances and one mass (Einstein, 1936). The magnification is $A(u)=\left(u^{2}+\right.$ $2) /\left[u^{2}\left(u^{2}+4\right)\right]^{1 / 2}$, where $u$ is the ratio $\theta / \theta_{E}$. Here, $\theta$ is the angular separation between the lens and the "true" position of the source (i.e. when the lens is far away), and $\theta_{E}$ is the natural angular scale for significant microlensing, $\theta_{E}=\left[4 G M / c^{2} \cdot(1-x) / L x\right]^{1 / 2}$, where $M$ is the lens mass, $L x$ its distance and $L$ the distance to the source $\rrbracket$. For small $u, A \simeq 1 / u$; at large $u, A$ dies out quite fast, $A \simeq 1+2 / u^{4}$.

As the lensing object moves with respect to the line of sight to the source star, microlensing phenomena are transient. Their natural timescale is the time needed to move by an angle equal to $\theta_{E}, t_{E}=$ $\theta_{E} / \mu$, where $\mu$ is the proper motion (angular velocity) of the lens w.r.t. the line of sight. The simplest microlensing light curves depend on four parameters, the baseline (unmagnified) flux, the time $t_{0}$ of maximum magnification $A_{\max }$, and the timescale $t_{E}$. If a significant fraction of

\footnotetext{
${ }^{1} \theta_{E}$ is also the angular radius of the so-called "Einstein" ring occuring for $\theta=0$.
} 
the flux comes from a blended star many $\theta_{E}$ away, a (fifth) blending parameter is necessary.

These four (five) parameters can be determined by fitting the known functional form of the light curve to the measurements. Of these parameters, only one contains information on the lens, $t_{E}$, and this is degenerate in the lens mass, distance and velocity. Two other parameters can be used, in a statistical sense, to test that the observed light curves are indeed due to microlensing : the source star magnitude and the minimum impact parameter $u_{0}\left(A_{\max }=A\left(u_{0}\right)\right)$. If one can ignore detection efficiency and blending effects, $u_{0}$ should display a flat distribution; its true expected distribution can be obtained from a simulation of the observing program. Similarly, as the lens does not choose the source it lenses, the source star should be distributed randomly in the colour-magnitude diagram, once again apart from detection efficiency which favors brighter stars. At present, such tests have only been done convincingly for microlensing in the galactic plane, where enough events have been found (few hundreds).

Ignoring efficiency, the average expected microlensing timescale for Magellanic Cloud stars is

$$
t_{E}=70 \text { days } \cdot\left(M / M_{\odot}\right)^{1 / 2} .
$$

(This is obtained from an "isothermal" spherical halo model that explains the galactic rotation curve, with $410^{11} M_{\odot}$ within $50 \mathrm{kpc}$.) The expected average $\theta_{E}$ is about 0.8 mas $\cdot\left(M / M_{\odot}\right)^{1 / 2}$, which explains why the search for microlensing has concentrated up to now on observing the magnification, not the deflection. Astrometric microlensing (i.e. measuring the photocentre motion during microlensing) will be feasible quite soon, especially with the foreseen FAME, GAIA and SIM astrometric satellites. I do not discuss it further and refer the reader to the review of Gould (2001); let me mention only that astrometric microlensing has a shallower dependence on the impact parameter, and that it provides information complementary to that of photometric microlensing; this will help in breaking the timescale degeneracy.

Apart from microlensing timescale, the other important observable is its rate. As $\theta_{E} \propto M^{1 / 2}$, a dark lens produces significant microlensing in a solid angle $\propto M$, and the probability that microlensing is currently occuring on a given star is thus proportional to the (weighted) integral of the mass density along the line of sight. This is known as the "optical depth" and it can be shown to be of order $\left(v_{\odot} / c\right)^{2}\left(v_{\odot}\right.$ is the galactic rotation velocity). This very low probability is the key factor for the observing strategy of the survey groups : they must monitor few tens of million stars a few times per week in order to reach the necessary sensitivity. Experimentally, the optical depth is measured from the 
detected microlensing events as

$$
\tau=\frac{\pi}{2} \frac{1}{N_{*} T_{\text {obs }}} \sum_{\text {evts }} \frac{t_{E}}{\epsilon\left(t_{E}\right)}
$$

where $N_{*}$ is the number of stars monitored for a duration $T_{o b s}$, and $\epsilon\left(t_{E}\right)$ is the overall detection efficiency for events with timescale $t_{E}$. 2

Finally, when blending is negligible or is due to a star with the same colour as that being microlensed, microlensing is achromatic. Survey groups thus monitor their fields in at least two bandpasses, using this as a tool to identify intrinsic variable stars, that are at least 10000 times more abundant than microlensing phenomena.

\subsection{General Microlensing}

In order to learn more about the lensing object, one needs more observables than just $t_{E}$. This is possible when the approximation of simple microlensing breaks down, and when another distance scale (perpendicular to the line of sight) can be measured and compared to $\theta_{E}$. There are three classes of such scales, according to whether they lie in the observer, source or lens plane.

In the observer plane, the Earth orbital motion can lead to measurable distortions that allow one to compare its semi-major axis to $\theta_{E}$ for long enough events, $t_{E}>3$ months (Gould, 1992). Such a "parallax" distortion is largest when the lens is closer to the observer, and unobservable when it is located near the source, in the Magellanic Clouds. Alternatively, the same microlensing phenomenon observed simultaneously from two distant points in the solar system could provide the same information (Refsdal, 1966) (see Soszyński et al. (2001) for a recent spectacular illustration of these). As they provide an additional distance scale in the observer plane, such situations allow one to measure the angular velocity of the lensing object as would be seen from the source star, $\mu_{M C}=\mu x /(1-x)$. This provides some discriminating power : Magellanic Cloud lenses will have a high MC-based angular velocity $\mu_{M C}$, whereas halo lenses will generally show a lower value.

In the source plane, the separation of the two components of a binary source star can play the same role, provided their revolution period is measured; examples are events ERos2-GSA2 (Derue et al., 1999) and MACHO-96-LMC-2 (Alcock et al., 2001b). Alternatively, the source star diameter can be used, especially when it nears or crosses a caustic curve (see e.g. Afonso et al., 2000 and references therein). In the case

\footnotetext{
${ }^{2}$ The definition of $\epsilon$ here is such that $\epsilon=100 \%$ if all microlensing events with $u_{0}<1$ occuring within $T_{\text {obs }}$ are detected.
} 
of a simple lens, the caustic "curve" reduces to a point, $\theta=0$. For a double lens, caustic curves have a more complicated structure, that can however be determined from the mesured light curve. If the source star angular diameter is known, the time it takes to cross the caustic curve is a rather direct measure of the relative proper motion of the lens, $\mu$, relative to the line of sight.

In the lens plane, finally, a double lens with measured separation and period would also allow to reduce, or even break the degeneracy.

Another interesting observable regarding double (or multiple) lenses is the relative rate of these with respect to simple microlensing. Double lenses can be detected as such when the component angular separation is of order $\theta_{E}$. When they are much closer, they act effectively as a single lens; when they are much further, one component is simply not detected from the light curve. The rate for detectable double lensing was estimated at 5-10\% for galactic plane lenses (Mao and Paczyński, 1991; Griest and $\mathrm{Hu}, 1992$ ). Recent measurements by OGLE (Udalski et al., 2000) bear out this prediction.

\section{Observations towards the LMC}

The Magellanic Clouds are close enough that many tens of million stars can be resolved from comparatively small ground-based telescopes, and far enough that a search for microlensing will sample an appreciable fraction of the galactic halo. Hence, they are presently the main targets in the search for machos. I first discuss observations towards the LMC.

Because they observed no microlensing candidate with a timescale shorter than 10 days, down to 0.04 day (1 hour), the EROs 1 and MACHO groups were able to exclude the possibility that more than $10 \%$ of the Galactic dark matter resides in planet-sized objects $\left(10^{-7}\right.$ to a few $\left.10^{-3} M_{\odot}\right)$. This resulted from a dedicated program by EROs1 (Aubourg et al., 1995; Renault et al., 1997; Renault et al., 1998), or as a byproduct of the search for heavier machos (Alcock et al., 1996). The two groups have combined their limits in Alcock et al. (1998).

In contrast, the first few events that were detected with longer timescales aroused at first high hopes that the Galactic dark matter problem was about to be solved. In the analysis of their first two-year data set (Alcock et al., 1997a), the MACHO group found 6-8 candidate events towards the LMC out of 7.5 million monitored stars. They estimated an optical depth of order half that required to account for the dynamical mass of a "standard" spherical dark halof; the typical timescale of the

\footnotetext{
${ }^{3}$ Slightly weaker limits were obtained for heavier machos of about $10^{-2} M_{\odot}$.

$44 \times 10^{11} \mathrm{M}_{\odot}$ within $50 \mathrm{kpc}$
} 
events, $t_{E}$, implied an average mass of about $0.5 M_{\odot}$ for halo lenses. The statistics was small enough that a halo full of machos was compatible with the result. Based on two candidates out of 3.3 million monitored stars, EROs1 preferred to set an upper limit on the halo mass fraction in objects of similar masses (Ansari et al., 1996; Renault et al., 1997). For typical brown dwarf masses, the limit was below that required to explain the rotation curve of our Galaxy, but it did not conflict with the MACHO result.

The first results towards the SMC then appeared (see next section). The EROs2 group published an even lower limit on machos, now excluding the upper part of the domain allowed by the MACHO results. It then seemed less likely that the halo could be fully comprised of $0.5 M_{\odot}$ machos.

The situation was partly clarified in 2000. The MACHO group presented an analysis of 5.7 year light curves of 10.7 million stars in the LMC (Alcock et al., 2000b) with an improved determination of their detection efficiency and a better rejection of background supernova explosions behind the LMC. A total of 13-17 microlensing candidates were found (including the previous 6 ). These two numbers refer to two different analyses of the same data, with stricter or looser selection criteria. These results from the MACHO group now favour a galactic halo macho component of $20 \%$ in the form of $0.4 M_{\odot}$ objects. This is their central value, but their result is compatible, at 95\% C.L., with a halo fraction ranging from 8 to $50 \%$ and a macho mass between 0.15 and $0.9 M_{\odot}$ (see Fig. 1). At about the same time, the detection of a halo white dwarf population at the level of a $10 \%$ component was also reported by Ibata et al. (2000).

Almost simultaneously, the EROS2 group presented its results from a two-year survey of 17.5 million stars in the LMC (Lasserre et al., 2000). One of the two EROs1 microlensing candidates was seen to vary again, 8 years after its first brightening, and was thus eliminated from the list of microlensing candidates. Two new candidates were identified. A few months later, this analysis was extended to three-year light curves of 25.5 million stars; this essentially confirmed the results of the twoyear analysis (Milsztajn et al., 2001). The total number of microlensing candidates towards the LMC from EROS is now five, one from EROS1 and four from EROs2. The candidates timescales agree with those from MACHO, at an average of about 30 days. Provided the events are due to halo lenses, this is compatible with $0.4 M_{\odot}$ lensing objects. The rate measured by EROs is about twice lower than that of MACHO. Because this rate is 10 times lower than expected if MACHOs are a substantial component of the galactic halo, and because the four new EROS candidates do not show excellent agreement with simple microlensing light 
curves, EROS2 continues to quote an upper limit on the fraction of the galactic halo in the form of MACHOs (see Fig. 1). It can be seen that this combined limit from all EROS data sets excludes the upper $25 \%$ of the parameter space domain favored by MACHO.

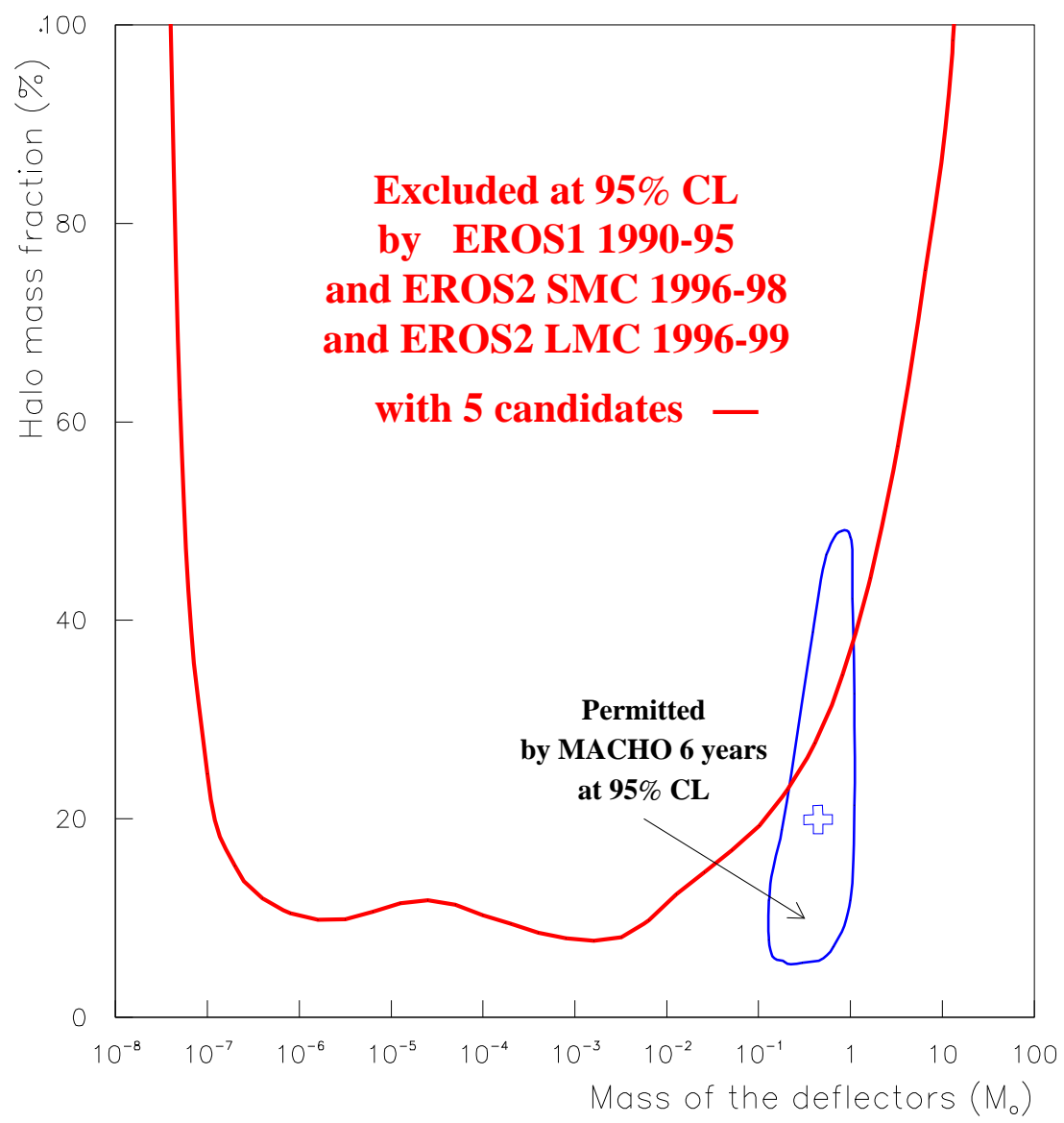

Figure 1. Results from EROS and MACHO on the macho component of the galactic halo, between 0 and $100 \%$ of a standard halo model $\left(4 \times 10^{11} M_{\odot}\right.$ inside a $50 \mathrm{kpc}$ radius sphere), for machos with masses ranging between $10^{-8}$ and $100 M_{\odot}$. The solid line is the $95 \%$ C.L. upper limit inferred by EROS from their five LMC microlensing candidates found in all LMC and SMC data, 1990-99 (Milsztajn et al., 2001). The closed contour is the MACHO 95\% C.L. accepted region, from their 13 LMC microlensing candidates found in 1992-98 (Alcock et al., 2000b). The preferred value is indicated by the cross.

The 13 MACHO microlensing candidates selected with stricter criteria all have timescales smaller than 75 days. The MACHO group has used this to obtain an upper limit on black hole dark matter in the galactic halo (Alcock et al., 2001a). They conclude that less than $40 \%$ of the 
halo dark matter can be in the form of 1 to $10 M_{\odot}$ objects. Altogether, microlensing surveys have now probed slightly more than 8 orders of magnitude in possible macho masses.

Some microlensing events towards the LMC display interesting peculiarities. Event MACHO-LMC-1 is much better fit by a binary lens with no caustic crossing (Dominik and Hirshfeld, 1996; Alcock et al., 2000a); this is presently the shortest timescale candidate towards the Magellanic Clouds. Event MACHO-LMC-9 is a double lens with caustic crossing (Alcock et al., 2000a); its proper motion is very low, thus favoring an interpretation as a double lens within the LMd ${ }^{5}$. The source star for event MACHO-LMC-14 is double (Alcock et al., 2001b) and this has allowed to conclude that the lens is in the LMC. Event MACHOLMC-5 is probably due to a galactic disk lens (Gould et al (1997) and D. Bennett, 2001, personal communication).

\section{Observations towards the SMC}

Although the SMC offers about 5-10 times less stars than the LMC for microlensing studies, it is a valuable target. Because the lines of sight to the LMC and SMC are close (about 20 degrees apart), the timescale distributions of microlensing candidates towards the two Clouds should be nearly identical if lenses belong to the galactic halo. (The difference in the average timescale for lenses of a given mass is much lower than the width of the distribution.) This point has been emphasised by Graff et al. (2001) - as reported in Milsztajn et al. (2001) - as a way to distinguish between microlensing being due primarily to halo lenses or to Magellanic Cloud lenses.

Also, the event rates should be comparable, although the ratio is more halo model dependent. Of course, this model dependency can be turned into an advantage, and may allow one to estimate the flattening of the halo (Sackett and Gould, 1993; Frieman and Scoccimarro, 1994; Alcock et al., 1995), if this is where the lenses are.

At the time of this review (June 2001), only two microlensing events have been detected towards the SMC, MACHO-97-SMC-1 (三 EROS-97SMC-1) and MACHO-98-SMC-1. These events imply an optical depth towards the SMC which is consistent with that measured towards the LMC by the MACHO group. (Because of the small statistics, this is not very informative.) However, further information exists on both events that point to microlensing due to SMC lenses.

The situation regarding the second microlensing event, MACHO-98SMC-1, is straightforward. It was first alerted upon by the MACHO group

${ }^{5}$ This view is not completely shared by Alcock et al. (2000a). 
on 25 May 1998, and a probable caustic crossing due to a double lens was announced soon after, on 8 June 1998. The source star was monitored by most microlensing groups. This allowed the second caustic crossing to be entirely covered, by the PLANET and EROS groups, resulting in a measurement of the crossing duration, from which the relative proper motion could be measured. Separate or combined analysis of the data from all five groups led to the conclusion that the double lens proper motion, about $1.4 \mathrm{~km} \mathrm{~s}^{-1} \mathrm{kpc}^{-1}$, is extremely unlikely to be that of a halo object (Afonso et al., 2000, and references therein). Therefore it must be located in the SMC.

The first candidate microlensing event towards the SMC was reported by Alcock et al. (1997b). It was observed independently by PalanqueDelabrouille et al. (1998). As observed by Palanque-Delabrouille et al. (1998) and confirmed by Udalski et al (1997), the source star is a periodic variable $(\mathrm{P}=5.12 \mathrm{~d} ; \mathrm{A}=5 \%)$. It is bright, actually the brightest for all microlensing events towards the Magellanic Clouds. It also has the longest timescale of all LMC or SMC events, $t_{E} \simeq 125 \mathrm{~d}$. As such, it is a particularly good place to look for a "parallax" distortion (see Sect. 2.2).

No parallax could be measured from EROs2 data, indicating that the lensing object is probably in the SMC (Palanque-Delabrouille et al., 1998). Graff et al. (2001) have also searched for a parallax distorsion

in the MACHO data for this event; this unsuccessful search produces a lower limit on $\mu_{M C}$ that strengthens this conclusion, at the $97 \%$ C.L.

The present situation is thus that none of the two microlensing events observed towards the SMC are due to halo lenses. The EROs2 group has used this to present limits obtained from their SMC two-year data alone on the fraction of stellar mass machos in the halo (Afonso et al., 1999).

\section{Discussion and Prospects}

I now discuss a few topics arising from these results in more detail.

\subsection{Statistical Tests}

The MACHO collaboration favours an interpretation of their candidates in terms of galactic halo lensing objects. They find that their sample is not, or little, contaminated by variable stars. In support of this, the distribution of stellar luminosities of their microlensing candidates agrees with that of LMC stars (Alcock et al., 2000b; Alcock et al., 2001c). The distribution of maximum magnifications is also compatible with a 
random minimum distance of approach of the lens to the star line of sight. These two tests could have revealed a possible contamination of the sample by intrinsic variable stars. (The same is true for the EROS candidates, but the small number of candidates precludes any significant conclusion.)

\subsection{MaChO vs. EROS : Optical Depth, Blending and EFFICIENCY}

Even if all EROS candidates are bona fide microlensing phenomena, the corresponding optical depth is twice lower than that from MACHO. As the two numbers are compatible within errors, this might simply be a statistical fluctuation. It seems useful, though, to search for candidates that should have been observed by both surveys. Such a study reveals no problem. The actual overlap between the two data sets is not very large, and only one candidate is found in both analyses : candidate EROS2-LMC-5 is identical to MACHO-LMC-26, which does not meet the MACHO strict selection criteria. Recall also that the first SMC microlensing event was observed by the two groups.

An accurate measurement of the optical depth requires knowledge of the number of monitored stars $N_{*}$, and unbiased estimates of the ratio $t_{E} / \epsilon\left(t_{E}\right)$ for all candidate events. Here, the effect of blending can be serious : it leads to an underestimate of $t_{E}$, and of $A_{\max }$ as well. (The latter can influence the candidate list through a minimum cut on this variable.) An underestimate of $t_{E}$ however can be partially compensated by the corresponding underestimate of the detection efficiency, $\epsilon$. On the other hand, faint stars blended with brighter ones can lead to additional detectable microlensing, for low enough impact parameters $u_{0}$. This can best be corrected for through simulated images. On that topic, the MACHO treatment is in principle more complete than that of EROS, and this could be an important point if the blending correction is large. But EROs fields are slightly less crowded in average, and studies have indicated that the overall effect of blending is small. Note that efficiencies from MACHO are larger than those of EROS; this depends a lot on the list of monitored stars, the sampling and on the chosen analysis cuts. A larger correction for blending in the EROS data would lead to a larger efficiency, and thus a lower microlensing optical depth, thus increasing the difference with MACHO.

Could it be that the different fields followed by EROS and MACHO really have different optical depths ? This would certainly be the case if most lenses are in the Magellanic Clouds, as the optical depth would then be larger towards the centre, where there are more stars, i.e. more lenses. The importance of this so-called "self-lensing" was first stressed 
in (Wu, 1994; Sahu, 1994). EROs covers a larger solid angle in the LMC $\left(64 \mathrm{deg}^{2}\right)$ than MACHO, which monitors primarily $15 \mathrm{deg}^{2}$ in the central part of the LMC. The EROS rate should thus be less contaminated by "self-lensing". However, the MACHO group has compared their sample to predictions of a specific model of the LMC, and finds that the observed distribution favours halo lenses, although they cannot completely exclude LMC lenses in a kind of LMC "halo" (Alcock et al., 2000b).

\subsection{LOCALISED LENSES}

Out of the 20 or so microlensing candidates towards the Magellanic Clouds, it has been possible to localise with reasonable certainty five of them. None of these localised lenses are in the halo. The two SMC events are due to SMC lenses (see Sect. 4), one from its binary lens nature, the other one from the absence of a measurable microlensing parallax. Three LMC event lenses have been localised, two in the LMC (one double lens, one double source) and one in the galactic disk.

The Magellanic Cloud double lenses (MACHO-98-SMC-1 and MACHOLMC-9) are especially interesting. If one naively extrapolates to Cloud lenses the result that, for galactic disk lenses, about $10 \%$ show their binarity in the light curve (Udalski et al., 2000), one is tempted to ask where are the $20(=10 \times 2)$ simple Magellanic Cloud lenses. Here again, the small statistics forbids us to go much further.

\subsection{More Statistics ?}

What are the prospects for more statistics towards the LMC ? The MACHO group has analysed 5.7 years of data out of a final total of 7.5 years (observations were terminated at the end of 1999). This would represent only a modest increase. In addition to the $15 \mathrm{deg}^{2}$ analysed in Alcock et al. (2000b), 25 more have been monitored with a less frequent sampling. These fields would have a lower efficiency for the range of $t_{E}$ displayed by present candidates, but would increase the sensitivity to heavier machos. The EROS group has analysed 3 years of data for 25 million stars, and expects to have a final data sample (by 2002) of 35 million stars over 6 years. This would represent an almost threefold increase in sensitivity. Also, the OGLE group has recently commissionned a new camera that will allow them to multiply their data taking rate by a factor 5; they will probably follow more Magellanic Clouds fields than in the past. 


\subsection{Comparing the LMC And SMC Candidates}

What are we to think of the present number of halo lenses (zero) in the search for microlenses towards the SMC ? In other words, how many SMC halo microlens events (with $t_{E}^{S M C} \simeq t_{E}^{L M C}$ ) should have been observed by both groups, if the signal presented by MACHO is entirely due to halo lenses ? Graff et al. (2001) have investigated this. The MACHO group monitored at least 2.2 million stars and has observed them over 6.5 years (Alcock et al. 1997b). Assuming equal detection efficiencies for SMC and LMC target stars, Graff et al. estimate that MACHO should observe in average $3.0 R$ events, where $R$ is the ratio of the optical depth towards the SMC and LMC. In the standard spherical halo model, $R=1.4$, and thus the expected number of events is about 4.2. In the same way, using the published EROs efficiencies towards the SMC (Afonso et al., 1999), one obtains for 6 years monitoring of 5.3 million stars about 3.6 events. Graff et al. estimate that, out of these $4.2+3.6=7.8$ events, only about 1.1 are common. Hence, provided none of the above assumptions is wrong, the prospect of an independent measurement of the optical depth towards the SMC looks good. Agreement in the microlensing rates and durations would represent, in my opinion, one of the most interesting tests of the halo lens hypothesis.

\subsection{Halo White Dwarfs}

What could the $0.4 M_{\odot}$ objects implied by the MACHO result be ? A possible answer is white dwarfs, although the mass seems a bit low. Many groups have undertaken the search for halo white dwarfs, that would be detected directly through their high proper motion when they traverse the galactic disk in the solar vicinity. A detection has been presented by Ibata et al. (2000), at the level of $10 \%$ of the expected local dark halo density. Other surveys report negative results (Flynn et al, 2001; Goldman, 2000). More recently, another detection was reported at the level of $2 \%$ of the expected local dark halo density (Oppenheimer et al., 2001). This result has been criticized by Graff (2001), Gibson and Flynn (2001), Reid et al. (2001) and Reyle et al. (2001). At present, it does not seem possible to identify these possible detections with the objects responsible for the MACHO result.

\section{Summary}

Microlensing surveys have not (yet ?) found enough machos to explain the galactic rotation curve. The undisputed result of the EROS and MACHO groups is that there exist strong limits on machos in the mass 
range between $10^{-7}$ and $10^{-1} M_{\odot}$. Between 0.1 and $1 M_{\odot}$, the two groups have candidates, some of which are undisputable microlensing phenomena. Their results are compatible, as is apparent in Fig. 1, but they interpret them differently. In my view, the issue of where the lenses are is not yet solved, but there are good prospects for doing so in the coming years. No long timescale candidates have been seen, that could be attributed to a halo object more massive than the Sun. Between 1 and $30 M_{\odot}$, the sensitivity of the two surveys is such that they should have already seen a few events due to putative heavy machos; the ensuing limits are still weak. Above $30 M_{\odot}$, there is not enough sensitivity yet. In the absence of alternative ideas to look for such heavy machos, any progress from the surveys in this mass range is welcome.

Finally, the search for microlensing towards the Magellanic Clouds will not stop with EROS and MACHO. Very soon, larger earth-based surveys, such as OGLE3 or SUPERMACHO, will also contribute to this topic. Microlensing towards M31 will provide a new line of sight that should help in interpreting the results. A few years later, simultaneous astrometric and photometric microlensing measurements from astrometric satellites will allow to directly find the lens position, for those few events with measurable "microlensing parallax".

\section{Acknowledgements}

I would like to thank D. Graff, T. Lasserre and J. Rich for their comments and careful reading of the manuscript.

\section{References}

Afonso C. et al. (ERos), 1999, A\&A 344, L63

Afonso C. et al. (ERos, macho-gman, mPs, ogle and Planet), 2000, ApJ 532, 340

Albrow M.D. et al. (PLANET), 2000, ApJ 535, 176

Alcock C. et al. (MACHO), 1993, Nat 365, 621

Alcock C. et al. (MACHO), 1995, ApJ 449, 28

Alcock C. et al. (MACHO), 1996, ApJ 471, 774

Alcock C. et al. (мACHO), 1997a, ApJ 486, 697

Alcock C. et al. (MACHO), 1997b, ApJL 491, L11

Alcock C. et al. (MACHO + EROs), 1998, ApJL 499, L9

Alcock C. et al. (MACHO + GMAN), 2000, ApJ 541, 270

Alcock C. et al. (МАСНO), 2000, ApJ 542, 281

Alcock C. et al. (MACHO), 2001a, ApJL 550, L169

Alcock C. et al. (мАСНО), 2001b, ApJ 552, 259

Alcock C. et al. (мАСHO), 2001c, ApJ 552, 582

Ansari R. et al. (EROs), 1996, A\&A 314, 94 
Arras P. and I. Wasserman, 1999, MNRAS 306, 257

Aubourg É. et al. (EROS), 1993, Nat 365, 623

Aubourg É. et al. (EROS), 1995, A\&A 301, 1

Aurière M. et al. (POINT-AGAPE), 2001, ApJL 553, L137

Bond I.A. et al. (MOA), 2001, preprint astro-ph/0102184, submitted to MNRAS

Derue F. et al. (EROS), 1999, A\&A 351, 87

Dominik M. and A. Hirshfeld, 1996, A\&A 313, 841

Einstein A., 1936, Science 84, 506 ; see also Renn J. et al., 1997, Science 275, 184, which show that the same result had already been obtained by Einstein in 1912

Flynn C. et al. 2001, MNRAS 322, 553

Frieman J. and R. Scoccimarro, 1994, ApJL 431, L23

Gibson B.K. and C. Flynn, 2001, Science 292, 2211, full text at www.sciencemag.org/cgi/content/full/2211a

Goldman B. (EROS), 2000, preprint astro-ph/0008383, to appear in the Proceedings of the Moriond 2000 workshop Cosmological physics with gravitational lensing.

Gould A., 1992, ApJ 392, 442

Gould A., J.N. Bahcall and C. Flynn, 1997, ApJ 482, 913

Gould A., 2000, preprint astro-ph/0004042, to appear in ASP conference series

Gould A., 2001, PASP 113, 903

Graff D., T. Lasserre and A. Milsztajn, 2001, in preparation

Graff D., 2001, Science 292, 2211, full text at www.sciencemag.org/cgi/content/full/2211a

Griest K. and W. Hu, 1992, ApJL 397, 362; ibid. 407, 440

Ibata R. et al., 2000, ApJ 532, L41

Lasserre T. et al. (EROS), 2000, A\&A 355, L39

Mao S. and B. Paczyński, 1991, ApJL 374, L37

Milsztajn A. et al. (ERos), 2001, Nucl. Phys. B (Proc. Suppl.) 91, 413

Oppenheimer B.R. et al., 2001, Science 292, 698

Paczyński B., 1986, ApJ 304, 1

Paczyński B., 1996, ARA\&A 34, 419

Palanque-Delabrouille N. et al. (EROS), 1998, A\&A 332, 1

Refsdal S., 1966, MNRAS 134, 315

Reid I., K. Sahu and S. Hawley, 2001, ApJ 559, 942

Reyle C., A. Robin and M. Creze, preprint astro-ph/0108134, to appear in A\&A

Renault C. et al. (EROs), 1997, A\&A 324, L69

Renault C. et al. (EROS), 1998, A\&A 329, 522

Rhie S.H. et al. (MPS + MOA), 2000, ApJ 533, 378

Roulet E. and S. Möllerach, 1997, Phys. Rep. 279, 67

de Rujula A., P. Jetzer and E. Masso, 1992, A\&A 254, 99

Sackett P. and A. Gould A., 1993, ApJ 419, 648

Sahu K.C., 1994, Nature 370, 275

Soszyński I. et al. (OGLE), 2001, ApJ 552, 731

Udalski A. et al. (OGLE), 1993, Acta Astron. 43, 289

Udalski A. et al. (OGLE), 1997, Acta Astron. 47, 431

Udalski A. et al. (OGLE), 2000, Acta Astron. 50, 1

Wu X.-P., 1994, ApJ 435, 66 